\title{
Safety and tolerability of intracerebroventricular PDGF-BB in Parkinson's disease patients
}

\author{
Gesine Paul, ${ }^{1}$ Olof Zachrisson, ${ }^{2}$ Andrea Varrone, ${ }^{3}$ Per Almqvist,,${ }^{2,4}$ Markus Jerling, ${ }^{2}$ Göran Lind, ${ }^{4}$ Stig Rehncrona, ${ }^{5}$ \\ Bengt Linderoth, ${ }^{4}$ Hjalmar Bjartmarz, ${ }^{5}$ Lisa L. Shafer, ${ }^{6}$ Robert Coffey, ${ }^{6}$ Mikael Svensson, ${ }^{4}$ Katarina Jansson Mercer, ${ }^{2}$ \\ Anton Forsberg, ${ }^{3}$ Christer Halldin, ${ }^{3}$ Per Svenningsson, ${ }^{7}$ Håkan Widner, Jonas Frisén, ${ }^{8}$ Sven Pålhagen, ${ }^{7}$ and Anders Haegerstrand ${ }^{2}$ \\ 'Department of Neurology, Department of Clinical Sciences, Lund University and Scania University Hospital, Lund, Sweden. ${ }^{2}$ Newron Sweden AB (former NeuroNova AB), Stockholm, Sweden. \\ ${ }^{3}$ Department of Clinical Neuroscience, Centre for Psychiatry Research, Karolinska Institutet, Stockholm, Sweden. ${ }^{4}$ Department of Neurosurgery, Karolinska University Hospital, Solna, Sweden. \\ ${ }^{5}$ Department of Neurosurgery, Lund University and Scania University Hospital, Lund, Sweden. ${ }^{6}$ Medtronic Inc., Minneapolis, Minnesota, USA. ${ }^{7}$ Department of Neurology, \\ Karolinska University Hospital, Huddinge, Sweden. ${ }^{8}$ Department of Cell and Molecular Biology, Karolinska Institutet, Stockholm, Sweden.
}

\begin{abstract}
BACKGROUND. Recombinant human PDGF-BB (rhPDGF-BB) reduces Parkinsonian symptoms and increases dopamine transporter (DAT) binding in several animal models of Parkinson's disease (PD). Effects of rhPDGF-BB are the result of proliferation of ventricular wall progenitor cells and reversed by blocking mitosis. Based on these restorative effects, we assessed the safety and tolerability of intracerebroventricular (i.c.v.) rhPDGF-BB administration in individuals with PD.
\end{abstract}

METHODS. We conducted a double-blind, randomized, placebo-controlled phase I/Ila study at two clinical centers in Sweden. Twelve patients with moderate PD received rhPDGF-BB via an implanted drug infusion pump and an investigational i.c.v. catheter. Patients were assigned to a dose cohort (0.2, 1.5, or $5 \mu \mathrm{g}$ rhPDGF-BB per day) and then randomized to active treatment or placebo (3:1) for a 12-day treatment period. The primary objective was to assess safety and tolerability of i.c.v.delivered rhPDCF-BB. Secondary outcome assessments included several clinical rating scales and changes in DAT binding. The follow-up period was 85 days.

RESULTS. All patients completed the study. There were no unresolved adverse events. Serious adverse events occurred in three patients; however, these were unrelated to rhPDGF-BB administration. Secondary outcome parameters did not show dose-dependent changes in clinical rating scales, but there was a positive effect on DAT binding in the right putamen.

CONCLUSION. At all doses tested, i.c.v. administration of rhPDGF-BB was well tolerated. Results support further clinical development of rhPDCF-BB for patients with PD.

TRIAL REGISTRATION. Clinical Trials.gov NCT00866502.

FUNDING. Newron Sweden AB (former NeuroNova AB) and Swedish Governmental Agency for Innovation Systems (VINNOVA).

\section{Introduction}

Parkinson's disease (PD) is the second most common neurodegenerative disease, affecting more than 5 million people worldwide. It is characterized by bradykinesia, rigidity, tremor, and gait disturbances, due to the progressive loss of midbrain dopaminergic neurons innervating the striatum. Even though pharmacological treatment can control symptoms for prolonged periods, treatment

\section{Related Commentary: p. 978}

Conflict of interest: Jonas Frisén was Director of Newron Sweden AB (former NeuroNova $A B)$, a fully owned subsidiary of Newron S.p.A., a listed company. Olof Zachrisson, Katarina Jansson Mercer, Per Almqvist, and Markus Jerling were employees of Newron Sweden AB. Anders Haegerstrand is an employee of Newron Sweden AB. Lisa L. Shafer and Robert Coffey have been employees of Medtronic Inc., which is a listed company. Role of funding source: NeuroNova AB acted as study sponsor, and VINNOVA provided funding.

Submitted: October 24, 2014; Accepted: January 9, 2015.

Reference information: / Clin Invest. 2015;125(3):1339-1346. doi:10.1172/JCI79635. and disease-related complications develop. There is an unmet clinical need for effective, long-lasting neuroprotective agents or restorative strategies to prevent further degeneration of nigrostriatal neurons and axons and to slow disease progression.

Several trophic and neurotrophic factors have been explored in animal models of PD (1), but only two neurotrophic factors (glial cell-derived neurotrophic factor [GDNF] and neurturin), both members of the GDNF family, have been explored in clinical trials, albeit with limited success $(2,3)$. In addition, the glucagonlike peptide-1 (GLP-1) agonist exendin-4 (exenatide) that also exhibits neuroprotective and neurogenic properties was investigated recently in an open-labeled, uncontrolled clinical study in patients with PD (4).

PDGFs are endogenous growth factors that occur in several different isoforms. The PDGF-B gene product forms the biologically active PDGF-BB dimer, which has been shown to have restorative effects in the dopaminergic system both in vitro and in vivo. PDGF improves the survival of embryonic tyrosine hydroxylase-positive 


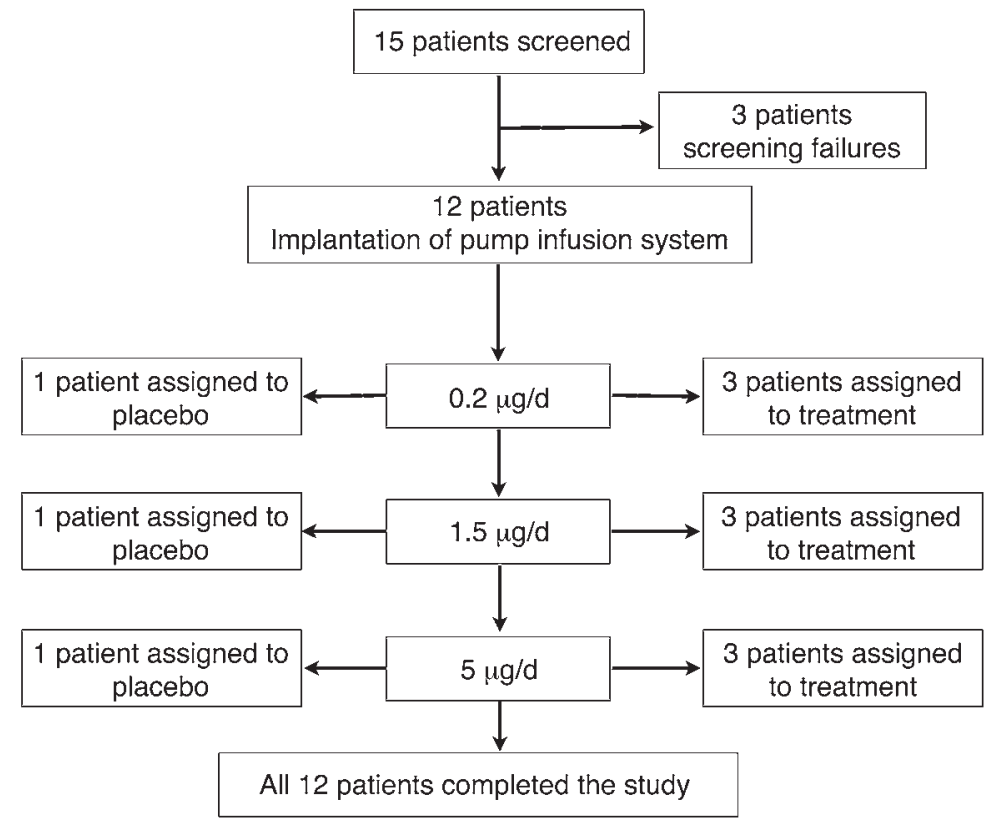

Figure 1. CONSORT flow diagram. For inclusion and exclusion criteria, see Supplemental Tables 1 and 2. For SAEs and AEs, see Tables 2 and 3, respectively.

neurons in vitro (5-7), and lesions in the mesostriatal dopaminergic system in vivo show evidence of elevated PDGF-B synthesis, which was interpreted as a possible endogenous neuroprotective response (8). Furthermore, recent animal studies demonstrated that intracerebroventricular (i.c.v.) administration of PDGF-BB for 2 weeks restored dopaminergic neurotransmission and provides functional recovery in several PD animal models. Injection of PDGF-BB resulted in (a) increased periventricular cell proliferation; (b) an increased number of tyrosine hydroxylase-positive dopaminergic neurons in the substantia nigra; (c) partial restoration of striatal dopamine transporter (DAT) levels; and (d) normalization of Parkinsonian behavior (9). The effect of PDGF-BB on dopaminergic neurons continued to develop after the treatment period and remained stable for several months after the end of treatment. Hence, the benefit of PDGF-BB does not appear to be the result of a direct pharmacological effect. Histologically, PDGF-BB leads to proliferation of SOX-2/Olig-1positive periventricular progenitor cells. Interestingly, all the effects of PDGF-BB on the nigrostriatal system were abolished if cell proliferation was blocked, suggesting that the effects of PDGF-BB are mediated by proliferating periventricular progenitor cells (9). There was no apparent generation of new dopaminergic cells in this experimental paradigm, and behavioral recovery was not achieved in a complete nigrostriatal lesion model. The exact restorative mechanism of PDGF-BB is uncertain; however, the preclinical data indicate that PDGF-BB-induced newborn progenitor cells have a trophic effect on remaining dopaminergic neurons and fibers (9).

The positive effects of PDGF-BB seen in preclinical models led us to evaluate PDGF-BB as a potential novel therapy for patients with PD. This first-in-human clinical study was conducted to investigate the safety and tolerability of recombinant human PDGF-BB (rhPDGF-BB) for i.c.v. administration. We also assessed the effects of i.c.v. rhPDGF-BB on motor and nonmotor symptoms as well as on striatal DAT binding, as measured by PET, with the
DAT radioligand $11 \mathrm{C}-\mathrm{N}$-(3-iodoprop-2 E-enyl)-2 $\beta$-carbomethoxy-3 $\beta$-(4-methylphenyl) nortropane $\left[{ }^{[1} \mathrm{C}\right] \mathrm{PE} 2 \mathrm{I}$ (referred to herein as $\left.\left[{ }^{11} \mathrm{C}\right] \mathrm{PE} 2 \mathrm{I}\right)$.

\section{Results}

\section{Patients}

Out of 15 patients screened, 12 patients with idiopathic PD of moderate severity were enrolled in the study. All 12 participants completed the study and were included in the final analysis (Figure 1).

Patients were divided into 3 cohorts according to dose level $(0.2,1.5$, or $5 \mu \mathrm{g}$ per day) and were enrolled in the study in a staggered fashion after review by the Independent Safety Monitoring Board (Figure 2). One patient in each cohort was randomized to placebo. The groups were comparable in terms of demographics, although the age range varied slightly among groups. The baseline demographic data for the 12 patients included in the study are presented in Table 1.

All patients received an intracranial catheter positioned in the lateral ventricle in the nondominant brain hemisphere connected to a subcutaneously implanted infusion pump for drug delivery. Patients received vehicle or PDGF-BB for 12 days and were followed for an additional 73 days, during which time they received an infusion of saline (Figure 3 ).

\section{Clinical outcomes}

Safety and tolerability. Adverse events (AEs) were reported for 11 of 12 patients. The most common AEs were pain and tissue reaction around the implanted pump. A small lacunar infarct in the right parietal lobe was noted in one patient in the $1.5 \mu \mathrm{g}$ per day dose group at follow-up, which was deemed unrelated to the treatment by the investigator. No pathological changes were identified based on assessments of patients' visual acuity or perimetry. There was no indication of weight loss or nausea in

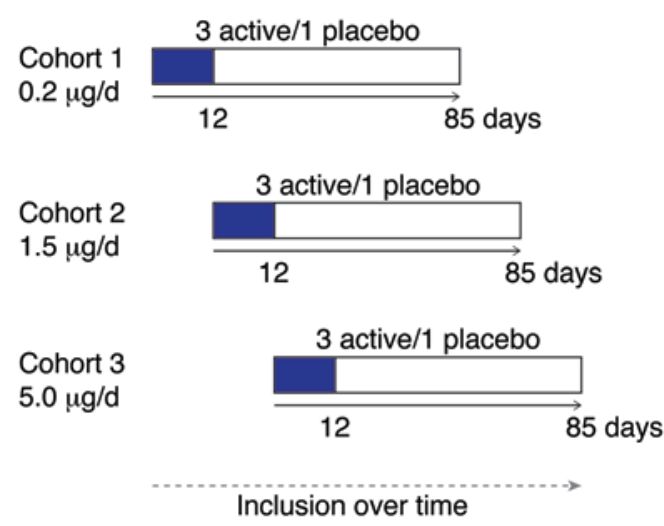

Figure 2. Experimental design. The study was designed as a dose-escalation study. The start of administration was staggered by at least 2 weeks between each patient. A Data Safety Monitoring Board approved the initiation of the second and third dose groups after all patients in the preceding cohort had completed week 3 of the study. Patients were monitored for a 10 -week follow-up period. For details of the investigational protocol, see Supplemental Table 3. 
Table 1. Demographics and baseline characteristics of the 12 participating patients

\begin{tabular}{|c|c|c|c|c|}
\hline & $\begin{array}{c}\text { Cohort } 1 \\
0.2 \mu \mathrm{g} / \mathrm{d}(n=3)\end{array}$ & $\begin{array}{c}\text { Cohort } 2 \\
1.5 \mu \mathrm{g} / \mathrm{d}(n=3)\end{array}$ & $\begin{array}{c}\text { Cohort } 3 \\
5 \mu \mathrm{g} / \mathrm{d}(n=3)\end{array}$ & $\begin{array}{l}\text { Placebo } \\
(n=3)\end{array}$ \\
\hline Mean age (yr [SD]) & $64( \pm 3)$ & $59( \pm 2)$ & $64( \pm 6)$ & $63( \pm 13)$ \\
\hline Mean age range (yr) & $61-70$ & $58-61$ & $58-70$ & $48-71$ \\
\hline Male $n(\%)$ & $2(66.7)$ & $2(66.7)$ & $2(66.7)$ & $1(33.3)$ \\
\hline Female $n(\%)$ & $1(33.3)$ & $1(33.3)$ & $1(33.3)$ & $2(66.7)$ \\
\hline Disease duration (yr [SD]) & $10( \pm 3)$ & $10( \pm 3)$ & $11( \pm 5)$ & $13( \pm 8)$ \\
\hline Disease duration range (yr) & $(8-13)$ & $(7-12)$ & $(5-15)$ & $(4-19)$ \\
\hline Hoehn \& Yahr stage & 2.66 & 2.66 & 2.33 & 2.66 \\
\hline LED (mean in mg [SD]) & $1,256( \pm 318)$ & $1,285( \pm 445)$ & $894( \pm 222)$ & $1,173( \pm 239)$ \\
\hline LED (range) & $964-1,595$ & $781-1,626$ & $752-1,150$ & $1,024-1,448$ \\
\hline
\end{tabular}

LED, levodopa equivalent dose.

except for a focal edema along the i.c.v. catheter before initiation of the infusion in a patient randomized to placebo. The event was assessed as related to the implantation procedure and resolved spontaneously. Another patient experienced a dislocation of the infusion pump caused by the rupture of sutures used to hold the pump in place, for which elective surgery to resuture the pump was performed. A third patient fractured her arm before implantation of the infusion system and required hospitalization. All 3 serious AEs (SAEs) were deemed to be unrelated to the study drug. There were no unresolved SAEs or other AEs at the end of the follow-up period at 85 days.

The position of the tip of the i.c.v. catheter was adequate in all patients and remained stable

the active treatment groups compared with the placebo-treated patients. There were no clinically significant abnormal findings for ECGs, vital signs, or clinical laboratory tests, except for one report of an increased mononuclear cerebrospinal fluid (CSF) cell count in one patient in the $0.2 \mu \mathrm{g}$ per day treatment group that resolved spontaneously. There were no signs of intracranial bleeding or loss of blood-brain barrier integrity based on bilirubin and albumin levels in the CSF.

All study participants underwent surgery according to the study protocol, and no unexpected surgical complications were noted during the study, as assessed by MRI examinations. The function of the SynchroMed II Programmable Infusion Pump was normal during the study (for a list and the frequency of SAEs and AEs, see Tables 2 and 3, respectively). No human PDGF-BB-specific antibodies were detected in any of the patients prior to drug administration or at follow-up (data not shown).

Secondary outcomes. The secondary objective of the study was to explore the effect of PDGF-BB on PD-related parameters, using validated clinical rating scales. These objectives were exploratory, since the study was neither designed nor powered to detect effi-
A

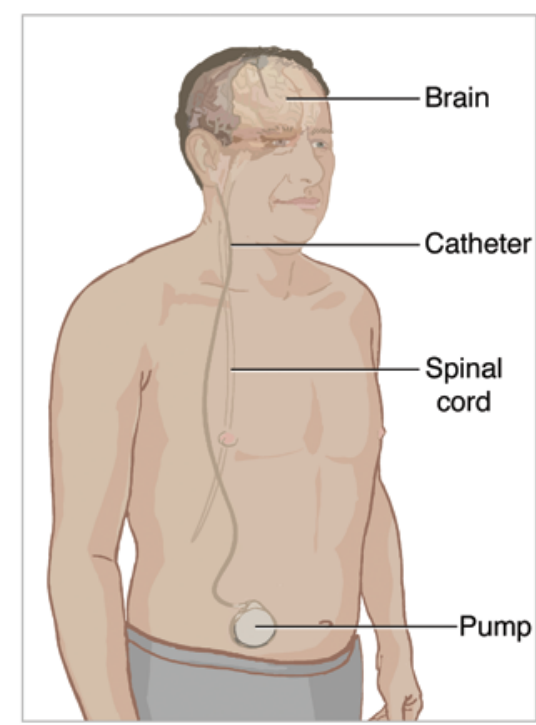

B

Infusion location

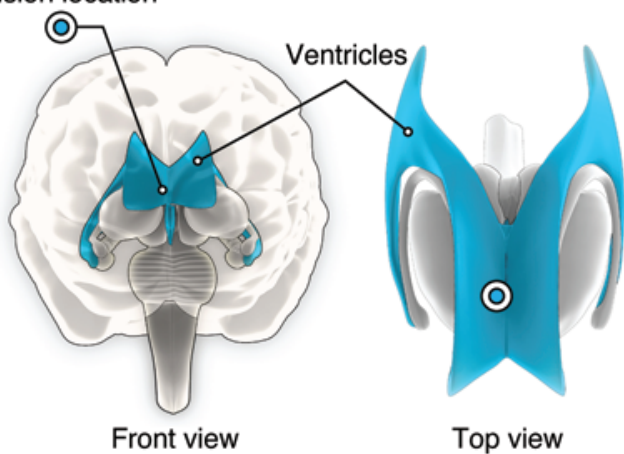

Front view
Top view

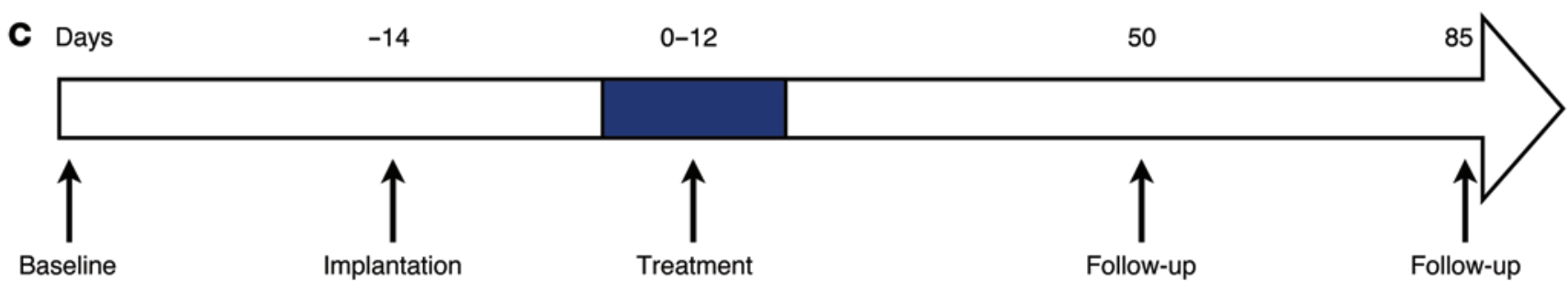

Figure 3. Delivery mode. (A) Patients were implanted with an abdominal drug delivery SynchroMed II pump connected to an i.c.V. catheter. (B) Front and top view of the lateral ventricular system (blue) in the human brain where PDCF-BB was administered via an implanted catheter, with its tip placed near the foramen of Monro. (C) Patients were examined at baseline, the catheter and pump were implanted, and the treatment with PDGF-BB or placebo was started within 14 days of surgery. 


\section{Table 2. Summary of SAEs}

$\begin{array}{lcccccc}\text { SAE no. } & \text { Treatment group } & \text { Reported event } & \text { Outcome } & \text { Seriousness } & \text { Relationship to study drug } & \text { Relationship to study device } \\ 1 & \text { Placebo } & \begin{array}{c}\text { Perifocal edema along the implanted } \\ \text { intracerebral catheter }\end{array} & \text { Resolved } & \text { Moderate } & \text { Not related } & \text { Related to implantation } \\ 2 & 5 \mu \mathrm{g} / \mathrm{d} & \text { Fracture left arm } & \text { Resolved } & \text { Moderate } & \text { Not related } & \text { Not related } \\ 3 & 5 \mu \mathrm{g} / \mathrm{d} & \text { Rupture of sutures for pump, with bleeding } & \text { Resolved } & \text { Moderate } & \text { Not related } & \text { Related to implantation }\end{array}$

cacy. PD motor-related symptoms were scored during an "off medication" state using the motor examination section (part III) of the Unified PD Rating Scale (UDPRS). At the end of study, all patients had a mean improvement of 4.5 points in the motor examination section of the UPDRS compared with baseline; however, this improvement was similar in all groups, including placebo-treated patients (Table 4 ).

The total Montgomery-Asberg depression rating scale (MADRS) scores indicated mild depression in 2 patients ( 1 in each of the $1.5 \mu \mathrm{g}$ per day and $5 \mu \mathrm{g}$ per day dose groups) at follow-up. All other patients had total MADRS scores that were not indicative of depression (Table 4). The total Mini-Mental State Examination (MMSE) scores indicated mild mental impairment (MMSE 22/30) in 1 patient in the $5 \mu$ g per day dose group at follow-up (Table 4). This patient already had a value in the lower range at baseline (MMSE 25/30) and later developed PD dementia.

Quality of life was assessed by using the EuroQoL-5D (EQ-5D) health state score and visual analogue scale. There was no change in quality of life in the treatment groups at follow-up compared with that at screening, whereas there was a slight improvement in the visual analogue scale in the placebo-treated group at follow-up compared with that at screening (Table 4).

\section{["'C]PE2I DAT binding}

$\left[{ }^{11} \mathrm{C}\right]$ PE2I DAT binding was assessed both prior to delivery of drug or vehicle and after the completion of the treatment, with time intervals ranging from 91 to 266 days between PET scans. All patients were scanned at least 14 days before treatment and $85 \pm$ 5 days after initiation of treatment. The time interval range between PET scans can be explained by logistical problems for the preoperative PET scan in relation to surgery and scheduled hospitalization for individual patients.

The most prominent effects of PDGF-BB treatment were seen in the right putamen, the region close to the tip of the drug delivery catheter and the one in which the highest concentration of PDGF-BB could be expected. Placebo-treated patients exhibited a reduction in mean $\left[{ }^{11} \mathrm{C}\right] \mathrm{PE} 2 \mathrm{I}$ DAT binding, whereas patients on active treatment ( $5 \mu \mathrm{g}$ per day) showed a mean increase (Figure 4$)$. A multiple regression analysis showed that the direc- tion and magnitude of change in $\left[{ }^{11} \mathrm{C}\right] \mathrm{PE} 2 \mathrm{I}$ DAT binding were independently related to the baseline value; the time interval between assessments and the PDGF-BB dose according to the regression function when applied to the observed change $=0.20-0.21 \times$ baseline value $-0.044 \times$ interval (months) $+0.068 \times$ dose $(\mathrm{mg})(P=$ 0.0061 for regression function with adjusted $r^{2}=0.68, P=0.011$ for baseline value, $P=0.036$ for time interval, and $P=0.0023$ for dose of PDGF-BB). The function shows that a high baseline value was associated with a more pronounced decrease, whereas the slope of change was shifted in a positive direction by the PDGF-BB treatment in a dose-dependent manner.

\section{Table 3. List and frequency of all AEs}

\begin{tabular}{|c|c|c|c|c|}
\hline AEs & $\begin{array}{c}\text { Placebo } \\
n(\%)\end{array}$ & $\begin{array}{c}0.2 \mu \mathrm{g} / \mathrm{d} \\
n(\%)\end{array}$ & $\begin{array}{c}1.5 \mu \mathrm{g} / \mathrm{d} \\
n(\%)\end{array}$ & $\begin{array}{c}5 \mu \mathrm{g} / \mathrm{d} \\
n(\%)\end{array}$ \\
\hline Device dislocation & $0(0.0)$ & $0(0.0)$ & $0(0.0)$ & $1(33.3)$ \\
\hline Implant site effusion & $0(0.0)$ & $1(33.3)$ & $0(0.0)$ & $0(0.0)$ \\
\hline Medical device pain & $1(33.3)$ & $1(33.3)$ & $1(33.3)$ & $0(0.0)$ \\
\hline Medical device site reaction & $1(33.3)$ & $2(66.7)$ & $0(0.0)$ & $0(0.0)$ \\
\hline Catheter site infection & $0(0.0)$ & $0(0.0)$ & $1(33.3)$ & $0(0.0)$ \\
\hline Macular degeneration & $0(0.0)$ & $0(0.0)$ & $0(0.0)$ & $1(33.3)$ \\
\hline Photopsia & $1(33.3)$ & $0(0.0)$ & $0(0.0)$ & $0(0.0)$ \\
\hline Nausea & $0(0.0)$ & $1(33.3)$ & $0(0.0)$ & $0(0.0)$ \\
\hline Herpes zoster & $0(0.0)$ & $1(33.3)$ & $0(0.0)$ & $0(0.0)$ \\
\hline Pneumonia & $1(33.3)$ & $0(0.0)$ & $0(0.0)$ & $0(0.0)$ \\
\hline Urinary tract infection & $1(33.3)$ & $0(0.0)$ & $0(0.0)$ & $0(0.0)$ \\
\hline Drug effect decreased & $0(0.0)$ & $0(0.0)$ & $1(33.3)$ & $0(0.0)$ \\
\hline Upper limb fracture & $0(0.0)$ & $0(0.0)$ & $0(0.0)$ & $1(33.3)$ \\
\hline Neck pain & $0(0.0)$ & $1(33.3)$ & $0(0.0)$ & $0(0.0)$ \\
\hline Seborrhoeic keratosis & $0(0.0)$ & $1(33.3)$ & $0(0.0)$ & $0(0.0)$ \\
\hline Dizziness & $0(0.0)$ & $1(33.3)$ & $0(0.0)$ & $0(0.0)$ \\
\hline Headache & $0(0.0)$ & $1(33.3)$ & $1(33.3)$ & $0(0.0)$ \\
\hline Hyperkinesia & $0(0.0)$ & $0(0.0)$ & $0(0.0)$ & $1(33.3)$ \\
\hline Lacunar infarction & $0(0.0)$ & $0(0.0)$ & $1(33.3)$ & $0(0.0)$ \\
\hline Migraine & $0(0.0)$ & $1(33.3)$ & $0(0.0)$ & $0(0.0)$ \\
\hline Pleocytosis & $0(0.0)$ & $1(33.3)$ & $0(0.0)$ & $0(0.0)$ \\
\hline Syncope & $1(33.3)$ & $0(0.0)$ & $0(0.0)$ & $0(0.0)$ \\
\hline Affect lability & $0(0.0)$ & $0(0.0)$ & $0(0.0)$ & $1(33.3)$ \\
\hline Hallucination & $1(33.3)$ & $0(0.0)$ & $0(0.0)$ & $0(0.0)$ \\
\hline Nephrolithiasis & $0(0.0)$ & $1(33.3)$ & $0(0.0)$ & $0(0.0)$ \\
\hline Actinic keratosis & $0(0.0)$ & $1(33.3)$ & $0(0.0)$ & $0(0.0)$ \\
\hline Eczema nummular & $0(0.0)$ & $1(33.3)$ & $0(0.0)$ & $0(0.0)$ \\
\hline Rash & $0(0.0)$ & $1(33.3)$ & $0(0.0)$ & $0(0.0)$ \\
\hline
\end{tabular}

The type of AEs, the number of patients ( $n$ ) reporting this event in each cohort, and the frequency of this event within the cohort expressed as a percentage are shown. 
Table 4. Secondary outcome parameter functional scores

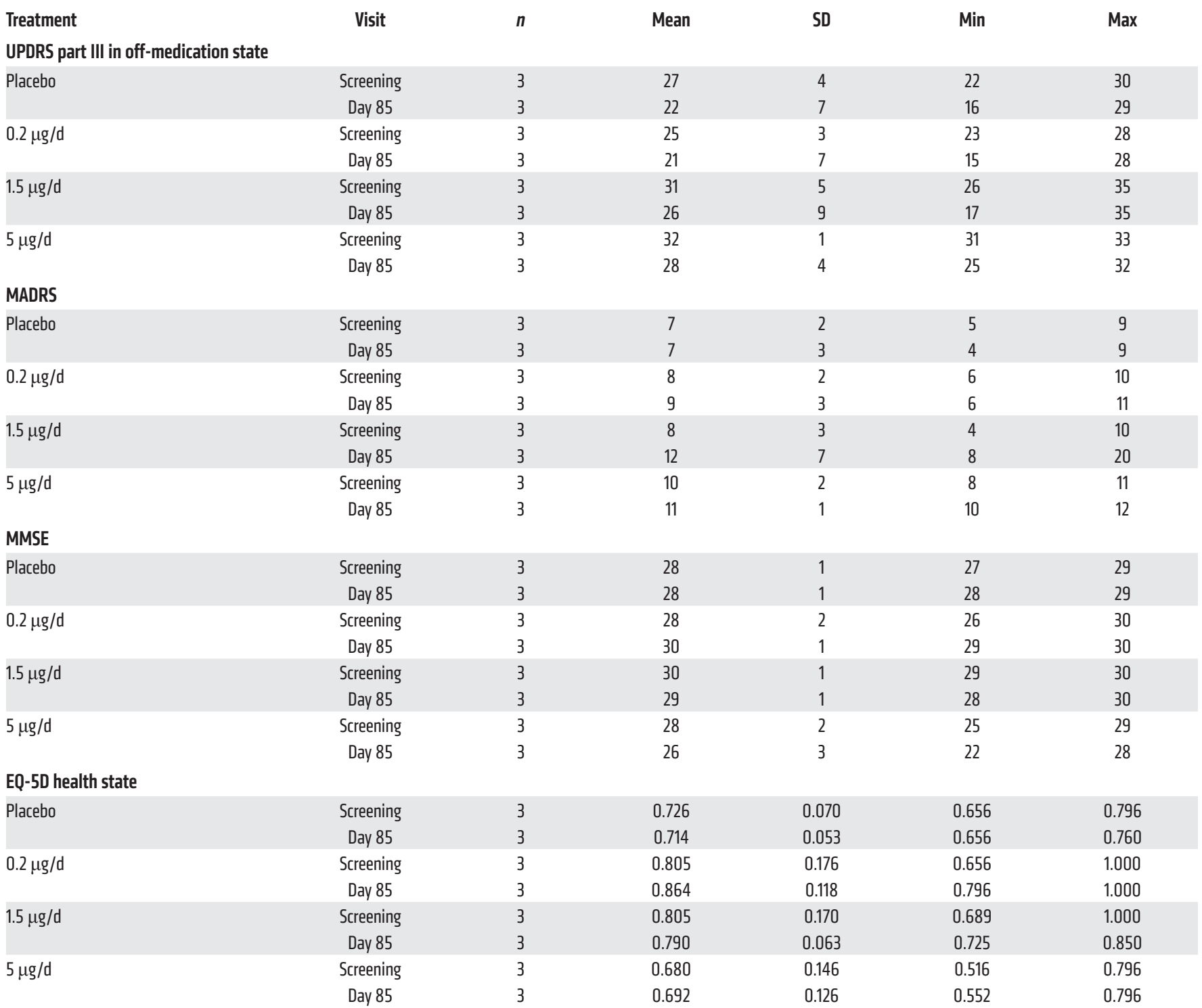

UPDRS part III evaluates motor dysfunction, and values are expressed from 0 to 60 . The MADRS evaluates depression and is interpreted as follows: 0-11, no depression; 12-20, mild depression; >20, high probability for true depression if the condition lasted for more than 2 weeks; $>40$, hospitalization at psychiatric clinic should be considered. The MMSE evaluates cognition, ranges from 0 to 30, and is interpreted as follows: $\geq 25-30$, normal; 21-24, mild; $10-20$, moderate; $\leq 9$, severe impairment. The EQ-5D health state examination evaluates quality of life and a score of 1.000 represents full health.

The changes in $\left[{ }^{11} \mathrm{C}\right]$ PE2I DAT binding in the analyzed regions over a period of 4 months, as predicted by the regression function, are shown in Table 5 and Supplemental Tables 4-6 (supplemental material available online with this article; doi:10.1172/ JCI79635DS1) for different combinations of baseline values and PDGF-BB doses. Absolute binding potentials of $\left[{ }^{11} \mathrm{C}\right]$ PE2I DAT binding are shown in Supplemental Table 7.

\section{Discussion}

Based on preclinical studies, neurotrophic factors offer promising therapy concepts for PD (1). However, only a few have reached the clinical testing phase, and the outcome of these clinical trials has generally been disappointing $(2,3)$. Difficulty in delivering the bioactive protein across the blood-brain barrier and tissue penetration appear to be the major limitations for the administration of neurotrophic factors targeting cells in the striatum or the substantia nigra. In contrast to other trophic factors investigated in PD, PDGF-BB targets stem and progenitor cells in the ventricular wall and thus constitutes a novel approach, with reduced demands for tissue penetration.

This is a first-in-human, double-blind, randomized, placebocontrolled phase I/IIa trial, reporting safety and tolerability of the i.c.v. delivery of rhPDGF-BB to patients with moderate PD using an investigational i.c.v. catheter and a SynchroMed II pump 


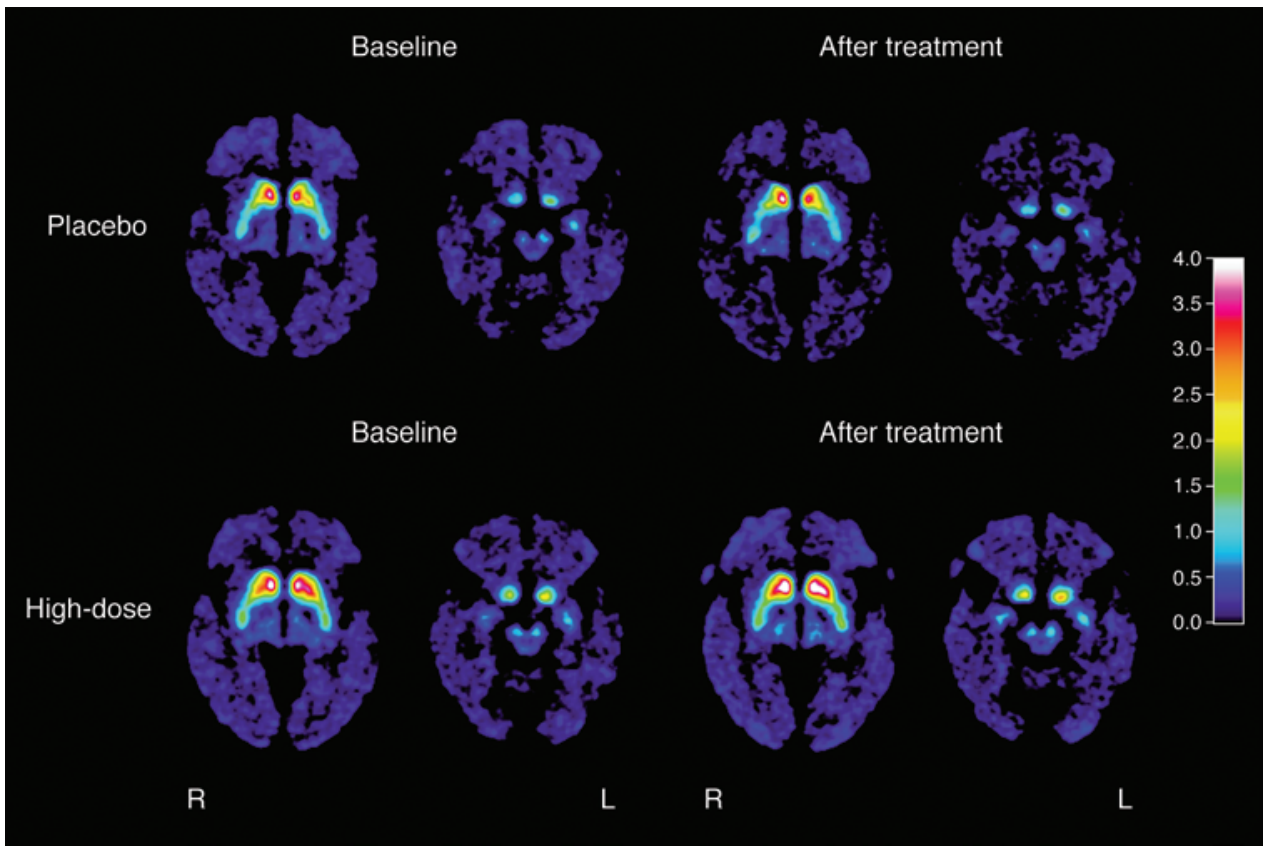

Figure 4. DAT binding. Mean parametric $\left[{ }^{[1} \mathrm{C}\right] \mathrm{PE} 2 \mathrm{I}$ images of binding potential $\left(B P_{\mathrm{ND}}\right)$ in the placebo $(n=3)$ and high-dose group $(n=3)$ at the level of the striatum and at the level of the substantia nigra. Images are displayed at baseline and after treatment with PDCF-BB.
$68 \%$ of the variability in change. With increasing PDGF-BB doses, the slope of change in binding shifted in a positive direction, and for the highest dose ( $5 \mu \mathrm{g}$ per day), the regression model, including all factors, predicted an absolute increase in binding over a time period of 4 months. This confirms similar findings in preclinical models, in which i.c.v. PDGF-BB infusion led to increased DAT binding in the striata of treated animals compared with that in controls (9). Even though the results of the PET investigations appear promising, one has to keep in mind that they are based on a small number of patients and cannot be generalized to a large population or to longer time periods. It still remains speculative whether the findings related to PDGF-BB in this study reflect a clinical improvement, and further clinical trials addressing efficacy in particular are warranted.

The current study provides data that will be useful for the design of future studies investigating the safety of PDGF-BB in patients with $\mathrm{PD}$, which should address a longer follow-up period, a further dose escalation, and repeated administration of PDGF-BB.

We conclude that a 2-week i.c.v. administration of PDGF-BB is safe and well tolerated in patients with moderate PD. In contrast to the potential benefit of PDGF-BB seen by the PET data, PDGF-BB did not show any dose-dependent effects on clinical efficacy parameters in this small sample size at the doses investigated. Further clinical trials are underway to study the efficacy of PDGF-BB and its potential to mediate long-term symptom relief as well as the effect of PDGF-BB on the restoration of the nigrostriatal system, as supported by preclinical studies, and its possible capacity to slow disease progression in PD.

\section{Methods} fore was not powered to detect any changes in efficacy parameters. However, we observed an improvement in UPDRS part III scores in all cohorts, including the placebo-treated patients. This is in line with a well-described placebo effect in PD trials, especially evident with complex and invasive interventions (10).

$\left[{ }^{11} \mathrm{C}\right]$ PE2I DAT binding, an indirect measure of the integrity and functional state of dopaminergic neurons, is arguably less influenced by a placebo effect compared with functional ratings and may provide an indication of a treatment effect on the nigrostriatal system. In line with longitudinal imaging studies of disease progression in $\mathrm{PD}$, in our study there was a generally more rapid decline in $\left[{ }^{11} \mathrm{C}\right]$ PE2I DAT binding in patients with higher baseline values (11). We found that the baseline value, the time interval between PET scans, and the PDGF-BB dose were independent and significant predictors of the absolute change in $\left[{ }^{11} \mathrm{C}\right]$ PE2I DAT binding; these factors combined explained

\section{Patients}

The study was conducted at two clinical centers in Sweden (Skåne University Hospital, Lund, and Karolinska University Hospital, Stockholm). Men and women aged 30 to 75 years, with a diagnosis of idiopathic moderate PD (Hoehn \& Yahr stage IIb-III) for longer than 5 years, were included.

Exclusion criteria were atypical or secondary parkinsonism, signs of dementia or depression, exposure to neuroleptic drugs within 6 months prior to the screening visit, a history of structural brain disease, and previous neurosurgical treatments of PD as well as any condition precluding a surgical procedure. Further details regarding inclusion and exclusion criteria are available in the Supplemental Information.

A Data Safety Monitoring Board analyzed the safety data and approved the commencement of treatment in each successive cohort. 


\section{Table 5. Change in ["C]PE2I DAT binding}

\begin{tabular}{|c|c|c|c|c|}
\hline Region & Dose in $\mu \mathrm{g} / \mathrm{d}$ & Baseline value & Change & $95 \% \mathrm{Cl}$ \\
\hline Right putamen & 0 & 0.5 & -0.080 & $-0.19,0.028$ \\
\hline \multirow[t]{11}{*}{$(P=0.0061)$} & & 1 & -0.19 & $-0.27,-0.11$ \\
\hline & & 1.5 & -0.29 & $-0.41,-0.18$ \\
\hline & 0.2 & 0.5 & -0.067 & $-0.17,0.040$ \\
\hline & & 1 & -0.17 & $-0.25,-0.096$ \\
\hline & & 1.5 & -0.28 & $-0.39,-0.17$ \\
\hline & 1.5 & 0.5 & 0.022 & $-0.082,0.13$ \\
\hline & & 1 & -0.084 & $-0.15,-0.017$ \\
\hline & & 1.5 & -0.19 & $-0.29,-0.091$ \\
\hline & 5 & 0.5 & 0.26 & $0.083,0.44$ \\
\hline & & 1 & 0.15 & $0.0032,0.30$ \\
\hline & & 1.5 & 0.048 & $-0.11,0.21$ \\
\hline \multirow{12}{*}{$\begin{array}{l}\text { Left putamen } \\
\text { (NS) }\end{array}$} & 0 & 0.5 & -0.045 & $-0.29,0.20$ \\
\hline & & 1 & -0.14 & $-0.33,0.05$ \\
\hline & & 1.5 & -0.23 & $-0.56,0.095$ \\
\hline & 0.2 & 0.5 & -0.037 & $-0.28,0.20$ \\
\hline & & 1 & -0.13 & $-0.31,0.053$ \\
\hline & & 1.5 & -0.22 & $-0.55,0.096$ \\
\hline & 1.5 & 0.5 & 0.016 & $-0.22,0.25$ \\
\hline & & 1 & -0.078 & $-0.23,0.079$ \\
\hline & & 1.5 & -0.17 & $-0.47,0.12$ \\
\hline & 5 & 0.5 & 0.16 & $-0.24,0.57$ \\
\hline & & 1 & 0.066 & $-0.27,0.41$ \\
\hline & & 1.5 & -0.029 & $-0.42,0.40$ \\
\hline
\end{tabular}

Change in [" $\left.{ }^{11}\right]$ PE2I DAT binding in the right and left putamen over 4 months by PDGF-BB dose and baseline binding value, as predicted by a regression model. Various other nigrostriatal regions are displayed in Supplemental Tables 4-6.

The board's analysis and decision were finalized 1 week after the 12-day treatment of the last patient in each of the two first cohorts. All patients, including placebo patients, were offered removal of the implanted device or participation in a follow-up and/or an openlabeled dose-escalation trial after the end of this study.

\section{Randomization and masking}

Patients were enrolled in a step-wise fashion to dose groups and then randomly assigned to receive treatment or placebo (vehicle) using a computer program, PROC PLAN in SAS. Patients, caregivers, treating neurologists, and neurosurgeons were blinded to the treatment allocation (active treatment or placebo), and all patients underwent the neurosurgical procedure to have the full infusion system implanted.

\section{Procedures}

Experimental design. Twelve patients were allocated to 1 of 3 dose cohorts $(0.2,1.5$, or $5 \mu \mathrm{g}$ rhPDGF-BB per day) and then randomized to active treatment or placebo (sodium acetate buffer) (3:1) (Figure 1). The start of treatment was staggered, with a minimum of 2 weeks between each patient (Figure 2). The pump was programmed for continuous infusion of study drug or placebo to achieve a daily dose of $0.2,1.5$, or $5 \mu \mathrm{g}$ per day of PDGF-BB or placebo for 12 days, after which all patients received sodium acetate buffer for 10 weeks until end of study.
Surgical procedure. All surgeries were performed under general anesthesia. MRI-guided stereotactic implantation (Leksell G-frame, Elekta) of a i.c.v. catheter (model 8770-4, Investigational Use i.c.v. Catheter, Medtronic) was performed via a burr hole on the coronal suture, 25 to $30 \mathrm{~mm}$ lateral to the midline on the right side, with the tip of the catheter in the lateral ventricle at the level of the foramen of Monro. The i.c.v. catheter was fixed to the skull bone with a custom anchor (Medtronic Inc.) at the burr hole. The position of the catheter was verified by intraoperative fluorography and by return of CSF from the catheter. A Medtronic SynchroMed II Programmable Infusion Pump (model 8637-20) was implanted subcutaneously in the right lower abdominal quadrant after programming and filling the pump reservoir with saline. The pump was connected to the i.c.v. catheter via a subcutaneously tunneled catheter. A postoperative cranial CT scan was carried out to ensure correct position of the catheter.

\section{PET imaging}

PET scans were performed at the Karolinska Institute, Stockholm, Sweden, at baseline and at the end of study (day 85). PET measurements were performed with the DAT radioligand $\left[{ }^{11} \mathrm{C}\right] \mathrm{PE} 2 \mathrm{I}$ using the HRRT system (Siemens Molecular Imaging). Each subject had an individual plaster helmet made to allow for repeated scans. A transmission scan of 6 minutes with a single source of ${ }^{137} \mathrm{Cs}$ was acquired immediately before radioligand injection. The radioligand was produced as described previously (12) and administered intravenously. List mode data were acquired for $93 \mathrm{~min}-$ utes, and images were reconstructed with a series of 31 frames of increasing duration (4 frames at $15 \mathrm{~s}, 4$ frames at $30 \mathrm{~s}, 6$ frames at $60 \mathrm{~s}, 6$ frames at $180 \mathrm{~s}$, and 11 frames at $360 \mathrm{~s}$ ). Images were reconstructed with 3D-OP-OSEM with PSF modeling as previously described (13). The in-plane resolution with this reconstruction method was $1.5 \mathrm{~mm}$ full with half maximum in the center of the field of view and $2.4 \mathrm{~mm}$ at $10 \mathrm{~cm}$ off center (13).

\section{Parametric mapping}

Parametric mapping of $\left[{ }^{11} \mathrm{C}\right]$ PE2I PET measurements was performed after motion correction as previously described (14). The wavelet-aided parametric imaging software (15) generated images of binding potential $\left(B P_{N D}\right)$, using the Logan noninvasive graphical analysis and the cerebellum as reference region. The $t^{*}$ (time of linearization) for the Logan graphical analysis was set at 54 minutes.

\section{Region-of-interest analysis}

The 3D-T1-weighted MRI of each patient was coregistered to the patient's PET images. Regions of interest were drawn on the caudate (head and body), putamen, ventral striatum, and substantia nigra. The regions of interest were then transformed to PET space and applied on the parametric images to obtain data for $B P_{\mathrm{ND}}$ for the respective region.

\section{Outcome parameters}

The primary objective was to assess safety and tolerability of i.c.v. administration of PDGF-BB and the device performance. Safety and tolerability were regularly monitored by ECGs; recording of vital signs; clinical laboratory tests; recording of AEs and withdrawals related to AEs; possible pathological changes in the brain, spinal cord, or retina identified through MRI; funduscopy; assessment of visual acuity and 
perimetry; and possible signs of intracranial bleeding or loss of bloodbrain barrier integrity in the CSF collected through lumbar puncture and device performance, as characterized by catheter tip placement (determined by MRI) and infusion accuracy (pump residual volume) (see detailed schedule in Supplemental Information).

Secondary objectives were to explore the effects of a 2-week treatment period with i.c.v. PDGF-BB on the time course of PD-related parameters, including disease severity, as assessed by the UPDRS; depressive symptoms, as assessed by the MADRS; cognition, as measured with the MMSE; and quality of life, as assessed with the EQ-5D rating scale. Other secondary outcome parameters included change in striatal DAT binding using PET scans of [11C]PE2I uptake at 3 months as compared with baseline, levels of PDGF-BB in the CSF collected through lumbar puncture (Quantikine Human PDGF-BB Kit, R\&D Systems, SBBO0, validated for hCSF by the study sponsor to detect levels $>250 \mathrm{pg} / \mathrm{ml}$ ), and the presence or absence of PDGF antibodies in the plasma (assay developed and validated by study sponsor, sensitivity 230-680 ng hIg/ml, using anti-PDGF-BB antibody from Millipore, catalog no. 07-1437, and secondary antibody from Southern Biotech, catalog no. 2010-05). For detailed PET, CSF, and plasma sampling schedules see Supplemental Information.

\section{Statistics}

AEs were summarized by incidence, frequency, and number of events. Scores of the UPDRS, EQ-5D, MADRS, and MMSE rating scales were summarized by treatment and time point and presented using descriptive statistics presenting the mean $\pm 1 \mathrm{SD}$ and range. For the PET analysis, longitudinal studies of disease progression in PD have demonstrated an exponential reduction in dopaminergic receptor populations over time, as measured by binding of ligands for DAT, VMAT2, and F-dopa (11, 16-19). Thus, baseline binding reflecting the stage of the disease is expected to have an impact on rate of change, with a more rapid decline from higher baseline values. The time interval between assessments will also affect the expected degree of change. Accordingly, change in $\left[{ }^{11} \mathrm{C}\right]$ PE2I DAT binding from before to after treatment was analyzed by multiple regression, with the following potential predictors: baseline value of binding, time interval between PET scans, and dose of PDGF-BB. A stepwise approach was used, with $P<0.05$ as the threshold for retaining parameters in the model.

\section{Study approval}

The study was approved by the Swedish Medical Product Agency and the local ethics committee in Stockholm, Sweden, as well as the Radiation Protection Committee, Karolinska Institute, Stockholm, Sweden, and performed in accordance with the Declaration of Helsinki and International Conference on Harmonization Good Clinical Practice guidelines. All patients provided informed written consent before enrollment.

\section{Acknowledgments}

The authors thank William Langston (The Parkinson's Institute) and Göran Rosenqvist and Martin Schain (PET Center, Karolinska Institutet). This work was supported by a grant from VINNOVA, the Swedish Governmental Agency for Innovation Systems.

Address correspondence to: Anders Haegerstrand, Newron Sweden AB, Södra Fiskartorpsvägen 15 C, SE-114 33 Stockholm, Sweden. Phone: 46.0.8.786.0900; E-mail: anders.haegerstrand@ newronsweden.com.
1. Rangasamy SB, Soderstrom K, Bakay RA, Kordower JH. Neurotrophic factor therapy for Parkinson's disease. Prog Brain Res. 2010;184:237-264.

2. Hickey P, Stacy M. AAV2-neurturin (CERE-120) for Parkinson's disease. Expert Opin Biol Ther. 2013;13(1):137-145.

3. Nutt JG, et al. Randomized, double-blind trial of glial cell line-derived neurotrophic factor (GDNF) in PD. Neurology. 2003;60(1):69-73.

4. Aviles-Olmos I, et al. Exenatide and the treatment of patients with Parkinson's disease. JClin Invest. 2013;123(6):2730-2736.

5. Nikkhah G, et al. Platelet-derived growth factor promotes survival of rat and human mesencephalic dopaminergic neurons in culture. Exp Brain Res. 1993;92(3):516-523.

6. Othberg A, Odin P, Ballagi A, Ahgren A, Funa K, Lindvall $O$. Specific effects of platelet derived growth factor (PDGF) on fetal rat and human dopaminergic neurons in vitro. Exp Brain Res. 1995;105(1):111-122.

7. Pietz K, Odin P, Funa K, Lindvall O. Protective effect of platelet-derived growth factor against 6-hydroxydopamine-induced lesion of rat dopaminergic neurons in culture. Neurosci Lett. 1996;204(1):101-104.
8. Funa K, et al. Enhanced synthesis of plateletderived growth factor following injury induced by 6-hydroxydopamine in rat brain. Neuroscience. 1996;74(3):825-833.

9. Zachrisson $\mathrm{O}$, et al. Restorative effects of platelet derived growth factor-BB in rodent models of Parkinson's disease. J Parkinsons Dis 2011;1(1):49-63.

10. Goetz CG, et al. Placebo influences on dyskinesia in Parkinson's disease. Mov Disord. 2008;23(5):700-707.

11. Schwarz J, Storch A, Koch W, Pogarell O, Radau PE, Tatsch K. Loss of dopamine transporter binding in Parkinson's disease follows a single exponential rather than linear decline. J Nucl Med. 2004;45(10):1694-1697.

12. Halldin C, et al. [(11)C]PE2I: a highly selective radioligand for PET examination of the dopamine transporter in monkey and human brain. Eur J Nucl Med Mol Imaging. 2003;30(9):1220-1230.

13. Varrone A, Sjoholm N, Eriksson L, Gulyas B, Halldin C, Farde L. Advancement in PET quantification using 3D-OP-OSEM point spread function reconstruction with the HRRT. Eur J Nucl Med Mol Imaging. 2009;36(10):1639-1650.

14. Schain M, et al. Quantification of serotonin trans- porter availability with [11C]MADAM--a comparison between the ECAT HRRT and HR systems. Neuroimage. 2012;60(1):800-807.

15. Cselenyi Z, Olsson H, Farde L, Gulyas B. Wavelet-aided parametric mapping of cerebral dopamine D2 receptors using the high affinity PET radioligand [11C]FLB 457. Neuroimage. 2002;17(1):47-60.

16. Brownell AL, Jenkins BG, Isacson O. Dopamine imaging markers and predictive mathematical models for progressive degeneration in Parkinson's disease. Biomed Pharmacother. 1999;53(3):131-140.

17. Hilker R, et al. Nonlinear progression of Parkinson disease as determined by serial positron emission tomographic imaging of striatal fluorodopa F 18 activity. Arch Neurol. 2005;62(3):378-382.

18. Nandhagopal R, et al. Longitudinal progression of sporadic Parkinson's disease: a multi-tracer positron emission tomography study. Brain. 2009;132(pt 11):2970-2979.

19. Kuriakose R, Stoessl AJ. Imaging the nigrostriatal system to monitor disease progression and treatment-induced complications. Prog Brain Res. 2010;184:177-192. 